\title{
Academic Honesty among Students of Selected Dental Colleges of Bangladesh
}

\author{
Sumi Gazi ${ }^{1}$, ABM Jamal ${ }^{2}$
}

\begin{abstract}
Objectives: The purpose of this research is to explore some of the issues related to academic honesty among students in Dental colleges, the challenges faced by them to do well academically in an honest manner.

Methods: This cross sectional study was conducted at ten public and private dental colleges of Bangladesh. The students were asked to fill up a self-administered, semi structured questionnaire containing about their Academic honesty and behavior. Data was collected using convenience sampling method. Sample size was 739.

Results: The study revealed 30\% marked proxy for their friends and $28.9 \%$ asked their friends to mark proxy for them. $10.8 \%$ cheated in examination using notes. Also $38.7 \%$ copied answers from another student during an examination.

Conclusion: The study revealed that a large number of dental students admitted to cheating and involvement in other forms of academic dishonesty.
\end{abstract}

Key words: Academic Honesty, Dental Students, Dental Colleges

\section{Introduction}

Impeccable moral and ethical values are desired attributes of a medical practitioner. Attitudes of dental students have an impact on the quality of health care provided to their patients. The basic personality of a person is strongly influenced by his or her attitudes and behavior (Asokan S, John B, Janani D, Jessy P, Kavya S \& Sharma K, 2013). Acts of academic dishonesty are common among university students. Academic institutions around the world have addressed this problem in many ways, generally by adopting rules, guidelines, and procedures to deal with it. Influences on a student's decision to commit improprieties in education may include pressure to succeed or pass a test, get better grades, or obtain specific benefits now or in the future. This behavior may originate in the family environment or culture.

The desired competencies and moral practices of medical professionals are outcomes of academic training. Honesty and integrity are fundamental virtues expected from medical student. However, deviation of academic honesty, which includes but is not limited to cheating, plagiarism and falsifying documentation, is not uncommon in medical colleges across the world. There is general agreement that there should be zero tolerance of cheating in a profession

\footnotetext{
1. Senior Lecurer, Medical Education Unit, and Hospital Coordinator, Sapporo Dental College and Hospital, Dhaka

2. Professor, Department of Surgery, Dhaka Medical College and Hospital, Dhaka
}

Address of correspondence:

Sumi Gazi, BDS, MSc, MMEd

Senior Lecurer, Medical Education Unit, and Hospital Coordinator Sapporo Dental College and Hospital, Dhaka

Email: sumigazi@yahoo.com based on trust and one on which human lives depend (Tanawattananacharoen S \& Nimnuan C, 2009).

\section{Methodology}

It was a descriptive type of cross sectional study. A total of 6 public and 4 private dental colleges were included in the study. They were Dhaka Dental College (DDC), Dental Unit of Sir Salimullah Medical College (SSMC), Dental Unit of Shaheed Suhrawardi Medical College (ShSMC), Dental Unit of Mymensingh Medical College (MMC), Bangladesh Dental College, Sapporo Dental College, Dental Unit of MH Samorita Medical College, Safena Women's Dental College, Update Dental College and Rangpur Dental College.

The study period was from July 2016 to June 2017 (one year). The study population was the students of $1^{\text {st }}, 2^{\text {nd }}, 3^{\text {rd }}$ and final year of selected 10 Dental Colleges. Total respondents were 739 students. Each of the 739 students were the sampling unit of this study. Convenience sampling technique was used. A self-administered semi structured questionnaire was used as research instrument. The students responded to all questions that requested an opinion by using a 5-point Likert scale, where $1=$ Strongly disagree, 2=Disagree, $3=$ Neither Agree Nor Disagree, 4=Agree, and $5=$ Strongly agree. All the collected data had been checked manually. Data editing, entry, processing and analysis were done using version 19 of SPSS. Interpretation of the qualitative data was presented by tables to compare the findings. There was a qualitative part of the questionnaire, to which none of the respondents responded. So it was left out of the data analysis.

Bangladesh Journal of Medical Education 2019;10(1):6-13. (C) 2019 Gazi et al., publisher and licensee Association for Medical Education. This is an Open Access article which permits unrestricted non-commercial use, provided the original work is properly cited. 


\section{Result}

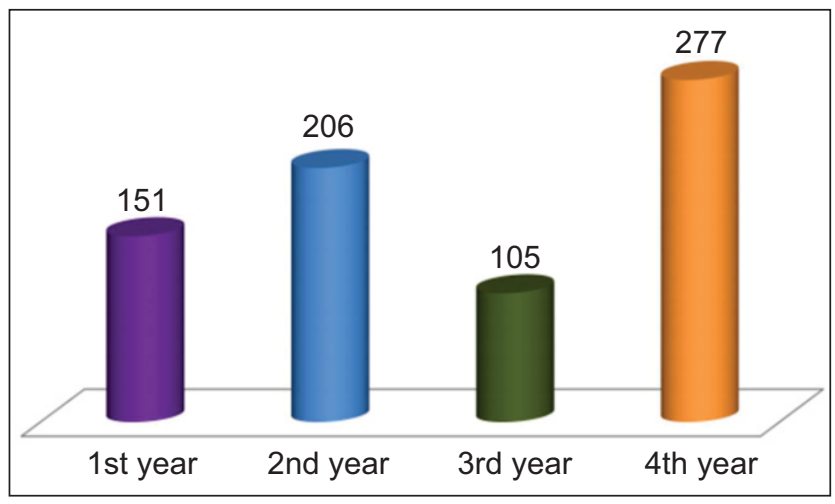

$\mathbf{n}=\mathbf{7 3 7}$

Figure 1: Distribution of the students by year

Figure 1 shows the distribution of the students by year. The total number of respondents was 739 . There were 151 respondents $(20.4 \%)$ in the 1 st year students. There were 206 respondents $(27.9 \%)$ in the 2 nd year students. There were 105 respondents $(14.2 \%)$ in the 3 rd year students. There were 277 respondents $(37.5 \%)$ in the 4 th year students.

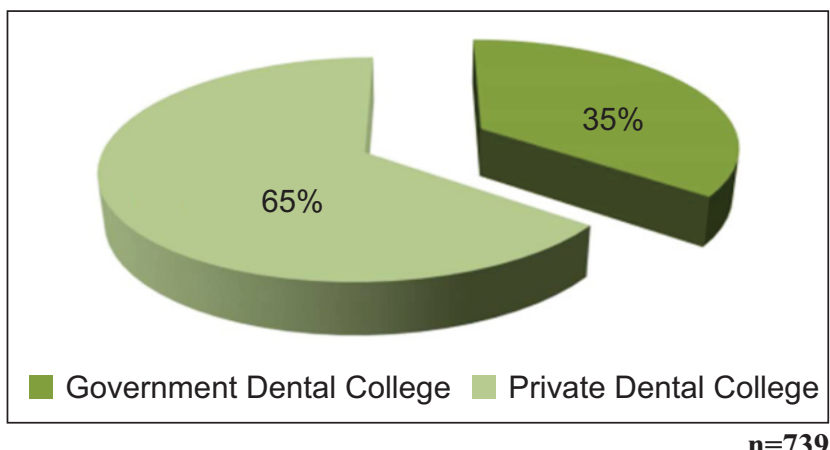

Figure 2: Distribution of students from the different types of Dental Colleges

Figure 2 shows the distribution of students from the different types of Dental Colleges. There were 261 respondents (35.3\%) from Public Dental Colleges and 478 respondents (64.7\%) from Private Dental Colleges.

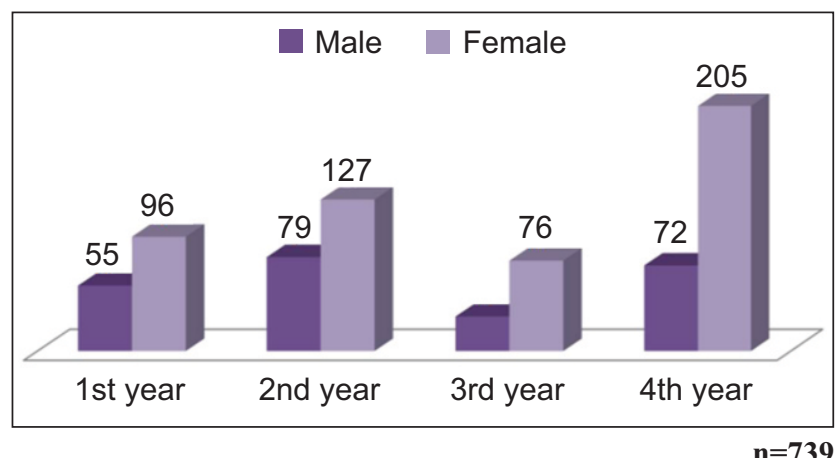

Figure 3: Distribution of respondents by gender and year of student
Figure 3 shows the distribution of respondents by Gender and Year of student. There were $235(31.8 \%)$ respondents who were male. From this there were 55 respondents who were from 1st year, 79 respondents who were from 2 nd year, 29 respondents who were from 3 rd year and 72 respondents who were from 4th year. There were 504(68.2\%) respondents who were female. From this there were 96 respondents who were from 1st year, 127 respondents who were from 2 nd year, 76 respondents who were from 3 rd year and 205 respondents who were from 4 th year.

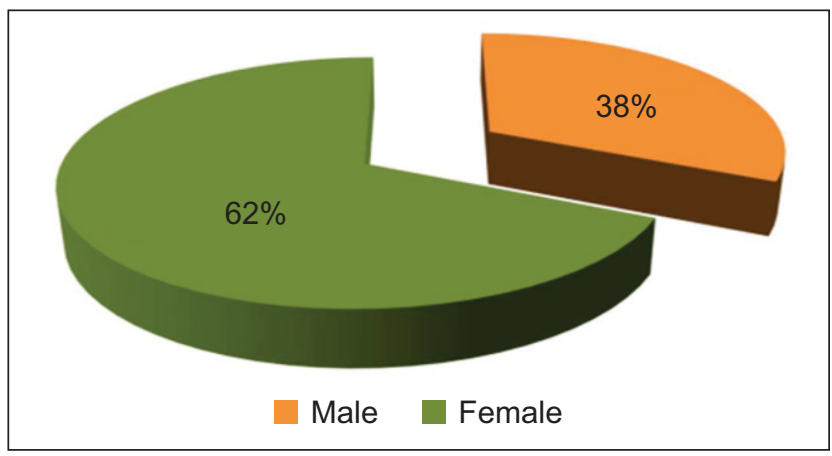

$\mathrm{n}=\mathbf{7 3 9}$

Figure 4: Distribution of the students by their gender

Figure 4 shows the distribution of respondents by Gender and Year of student. There were $235(31.8 \%)$ respondents who were male. There were 504(68.2\%) respondents who were female.

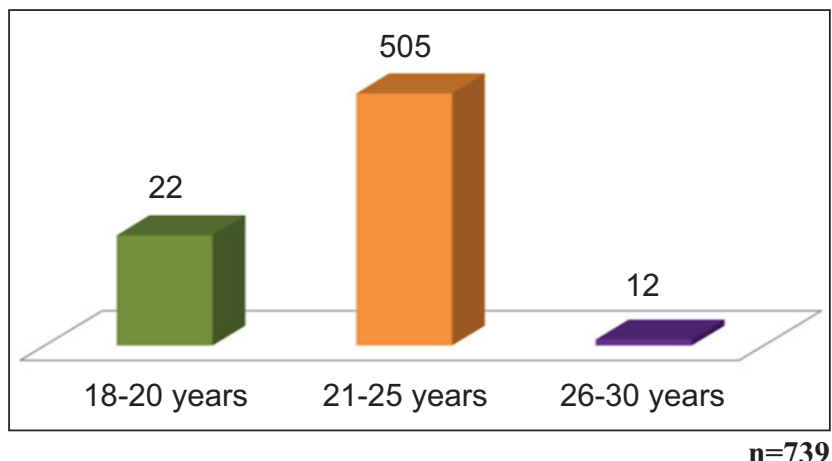

Figure 5: Distribution of the students by their Age

Figure 5 shows the distribution of the students by age in the 739 respondents. There were 222 respondents who were from 18-20 years, there were 505 respondents who were from 21-25 years and there were 12 respondents who were from 26-30 years old 


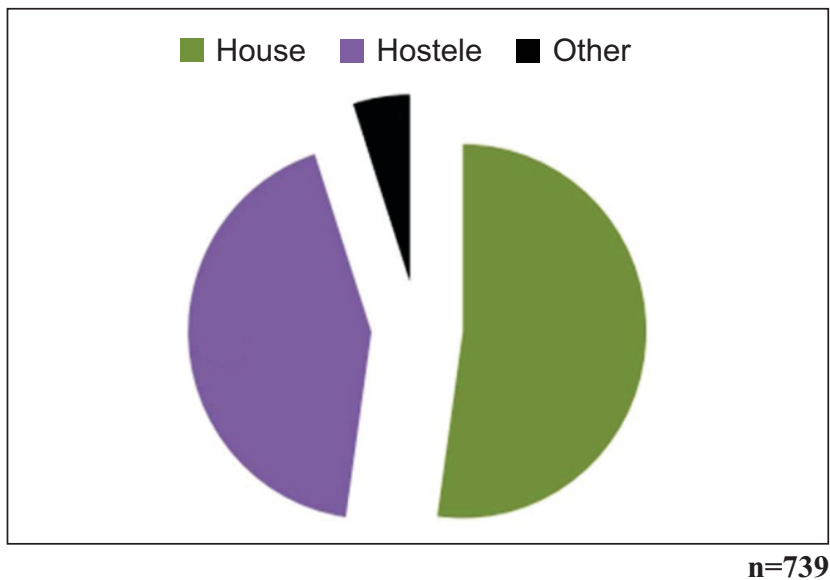

Figure 6: Distribution of the student by their residence

Figure 6 shows the distribution of the students by their residence in the 739 respondents. There were $386(52 \%)$ respondents who were living in houses, there were 316 (42.8) respondents who were living in hostel and there were $37(5.0 \%)$ respondents who were living in other type of accommodation.

Key to Tables: Level of agreement:

1. SA (Strongly Agree): When respondent largely agrees with the statement

2. A (Agree): When respondent has some agreement with the statement

3. NAND (Neither Agree Nor Disagree): When respondent cannot agree or disagree with the statement

4. D (Disagree): When respondent has some disagreement with the statement

5. SD (Strongly Disagree): When respondent largely disagrees with the statement

Table 1: Distribution of the respondents' opinions about deviation from practising academic honesty regarding attendance

\begin{tabular}{|l|c|c|c|c|c|c|}
\hline \multirow{4}{*}{ Statement } & $\mathbf{5}$ & $\mathbf{4}$ & $\mathbf{3}$ & $\mathbf{2}$ & $\mathbf{1}$ & Total \\
\cline { 2 - 7 } & $\begin{array}{c}\text { SA } \\
\mathbf{f}(\mathbf{\%})\end{array}$ & $\begin{array}{c}\mathbf{A} \\
\mathbf{f}(\%)\end{array}$ & $\begin{array}{c}\text { NAND } \\
\mathbf{f}(\%)\end{array}$ & $\begin{array}{c}\mathbf{D} \\
\mathbf{f}(\mathbf{\%})\end{array}$ & $\begin{array}{c}\text { SD } \\
\mathbf{f}(\mathbf{\%})\end{array}$ & \\
\hline $\begin{array}{l}\text { I stay in the } \\
\text { dental college } \\
\text { during } \\
\text { academic } \\
\text { hours }\end{array}$ & 335 & 269 & 33 & 32 & 65 & 734 \\
$(45.6 \%)$ & $(36.6 \%)$ & $(4.5 \%)$ & $(4.4 \%)$ & $(8.9 \%)$ & \\
\hline $\begin{array}{l}\text { I have left } \\
\text { before class } \\
\text { finished } \\
\text { without } \\
\text { permission }\end{array}$ & $\begin{array}{c}26 \\
(3.5 \%)\end{array}$ & $\begin{array}{c}97 \\
(13.2 \%)\end{array}$ & $\begin{array}{c}38 \\
(5.2 \%)\end{array}$ & $\begin{array}{c}256 \\
(34.7 \%)\end{array}$ & $\begin{array}{c}320 \\
(43.4 \%)\end{array}$ & 737 \\
\hline
\end{tabular}

Table 1 shows practising academic honesty regarding attendance. The highest percentage responding is $39.6 \%$ strongly disagreed (SD) for asking signing a friend to sign proxy attendance and $35.2 \%$ strongly disagreed (SD) for signing proxy attendance at for a friend who did not attend the class. The percentage of those who strongly agreed (SA) was $7.6 \%$, and agreed (A) was $21.3 \%$ to asking a friend to sign proxy attendance. Similarly percentage of those who strongly agreed (SA) was $8.1 \%$, and agreed (A) was $21.9 \%$ to have signed proxy for a friend.

Table 2. Distribution of the respondents' opinions about deviation from practising academic honesty regarding justifying their absence from class

$\mathbf{n}=\mathbf{7 3 9}$

\begin{tabular}{|l|c|c|c|c|c|c|}
\hline \multirow{2}{*}{ Statement } & $\mathbf{5}$ & $\mathbf{4}$ & $\mathbf{3}$ & $\mathbf{2}$ & $\mathbf{1}$ & Total \\
\cline { 2 - 7 } & $\begin{array}{c}\text { SA } \\
\mathbf{f ( \% )}\end{array}$ & $\begin{array}{c}\mathbf{A} \\
\mathbf{f}(\%)\end{array}$ & $\begin{array}{c}\text { NAND } \\
\mathbf{f ( \% )}\end{array}$ & $\begin{array}{c}\mathbf{D} \\
\mathbf{f ( \% )}\end{array}$ & $\begin{array}{c}\text { SD } \\
\mathbf{f}(\mathbf{\%})\end{array}$ & \\
\hline $\begin{array}{l}\text { I have faked } \\
\text { illness to } \\
\text { justify my } \\
\text { absence }\end{array}$ & $\begin{array}{c}25 \\
(3.4 \%)\end{array}$ & $\begin{array}{c}99 \\
(13.5 \%)\end{array}$ & $\begin{array}{c}51 \\
(6.9 \%)\end{array}$ & $\begin{array}{c}194 \\
(26.4 \%)\end{array}$ & $\begin{array}{c}365 \\
(49.7 \%)\end{array}$ & 734 \\
\hline $\begin{array}{l}\text { I sometimes } \\
\text { remain absent } \\
\text { for classes } \\
\text { without any } \\
\text { genuine cause }\end{array}$ & $\begin{array}{c}54 \\
(7.4 \%)\end{array}$ & $\begin{array}{c}196 \\
(26.7 \%)\end{array}$ & $\begin{array}{c}57 \\
(7.8 \%)\end{array}$ & $\begin{array}{c}210 \\
(28.6 \%)\end{array}$ & $\begin{array}{c}217 \\
(29.6 \%)\end{array}$ & 739 \\
\hline $\begin{array}{l}\text { I have } \\
\text { submitted } \\
\text { fake medical } \\
\text { certificate to } \\
\text { justify illness }\end{array}$ & $\begin{array}{c}(3.1 \%) \\
(7.3 \%)\end{array}$ & $\begin{array}{c}54 \\
(3.7 \%)\end{array}$ & $\begin{array}{c}(23.7 \%) \\
(62.1 \%)\end{array}$ & 737 \\
\hline
\end{tabular}

Table 2 shows practising academic honesty regarding justifying their absence from class. The highest percentage of academic honesty is $62.1 \%$ strongly disagreed (SD) for submitting fake medical certificate to justify illness. Those responding by strongly agreeing (SA) was 3.4\% and agreeing (A) was $13.5 \%$ to faking illness. Those responding by strongly agreeing (SA) was $7.4 \%$ and agreeing (A) was $26.7 \%$ to remain absent without genuine cause. Those responding by strongly agreeing (SA) was 3.1\% and agreeing (A) was 7.3\% to submitting fake medical certificate to justify illness.

Table 3: Distribution of the respondents' opinions about deviation from practising academic honesty regarding presence in class and in college during academic hours

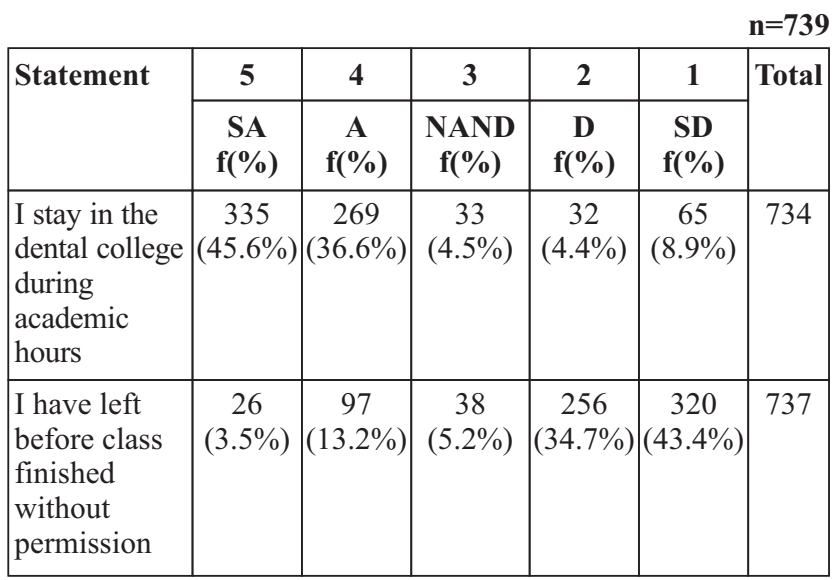


Table 3: Shows practising academic honesty regarding presence in class and in college during academic hours. Those responding by strongly agreeing (SA) was $45.6 \%$ and agreeing (A) was $36.6 \%$ to staying in the dental college during academic hours. Those responding by strongly agreeing (SA) was $3.5 \%$ and agreeing (A) was $13.2 \%$ to leaving before class finished without permission.

Table 4: Distribution of the respondents' opinions about deviation from practising academic honesty regarding Assignments

\begin{tabular}{|c|c|c|c|c|c|c|}
\hline & & & & & & $n=739$ \\
\hline Statement & 5 & 4 & 3 & 2 & 1 & Total \\
\hline & $\begin{array}{c}\text { SA } \\
\mathbf{f}(\%)\end{array}$ & $\begin{array}{c}\text { A } \\
\mathbf{f}(\%)\end{array}$ & $\begin{array}{c}\text { NAND } \\
\mathbf{f}(\%)\end{array}$ & $\begin{array}{c}D \\
f(\%)\end{array}$ & $\begin{array}{c}\text { SD } \\
\mathbf{f}(\%)\end{array}$ & \\
\hline $\begin{array}{l}\text { For an } \\
\text { assignment, I } \\
\text { have copied } \\
\text { verbatim } \\
\text { (word-for- } \\
\text { word) from } \\
\text { the internet, } \\
\text { textbooks, } \\
\text { journals, } \\
\text { papers and } \\
\text { not listed } \\
\text { them as } \\
\text { references }\end{array}$ & $\begin{array}{c}52 \\
(7.1 \%)\end{array}$ & $\begin{array}{c}313 \\
(43 \%)\end{array}$ & $\begin{array}{c}110 \\
(15.1 \%)\end{array}$ & $\begin{array}{c}138 \\
(19 \%)\end{array}$ & $\begin{array}{c}115 \\
(15.8 \%)\end{array}$ & 728 \\
\hline $\begin{array}{l}\text { I have copied } \\
\text { answers form } \\
\text { another } \\
\text { student's work } \\
\text { for an } \\
\text { assignment }\end{array}$ & $\begin{array}{c}31 \\
(4.2 \%)\end{array}$ & $\begin{array}{c}246 \\
(33.4 \%)\end{array}$ & $\begin{array}{c}87 \\
(11.8 \%)\end{array}$ & $\begin{array}{c}183 \\
(24.9 \%)\end{array}$ & $\begin{array}{c}189 \\
(25.7 \%)\end{array}$ & 736 \\
\hline $\begin{array}{l}\text { For an } \\
\text { assignment I } \\
\text { have copied } \\
\text { form } \\
\text { assignments } \\
\text { submitted } \\
\text { earlier by } \\
\text { senior peers }\end{array}$ & $\begin{array}{c}45 \\
(6.1 \%)\end{array}$ & $\begin{array}{c}234 \\
(31.9 \%)\end{array}$ & $\begin{array}{c}101 \\
(13.8 \%)\end{array}$ & $\begin{array}{c}199 \\
(27.1 \%)\end{array}$ & $\mid \begin{array}{c}155 \\
(21.1 \%)\end{array}$ & 734 \\
\hline
\end{tabular}

Table 4 shows deviation from practising academic honesty regarding assignments. The percentage of deviation from academic honesty is $7.1 \%$ strongly agreeing (SA) and $43 \%$ agreeing (A) to practising plagiarism. $4.2 \%$ strongly agreeing (SA) and 33.4\% agreeing (A) to have copied answers from another student's work for an assignment. 6.1\% have strongly agreed (SA) and $31.9 \%$ have agreed (A) to have copied from assignments submitted earlier by senior peers.
Table 5: Distribution of the respondents' opinions about deviation from practising academic honesty regarding Written Work

$\mathbf{n}=\mathbf{7 3 9}$

\begin{tabular}{|c|c|c|c|c|c|c|}
\hline \multirow[t]{2}{*}{ Statement } & 5 & 4 & 3 & 2 & 1 & \multirow[t]{2}{*}{ Total } \\
\hline & $\begin{array}{c}\text { SA } \\
\mathbf{f}(\%)\end{array}$ & $\begin{array}{c}\mathbf{A} \\
\mathbf{f}(\%)\end{array}$ & $\begin{array}{c}\text { NAND } \\
\text { f(\%) }\end{array}$ & $\begin{array}{c}\text { D } \\
\mathbf{f}(\%)\end{array}$ & $\begin{array}{c}\text { SD } \\
\mathbf{f}(\%)\end{array}$ & \\
\hline $\begin{array}{l}\text { I have } \\
\text { modified } \\
\text { another } \\
\text { student's } \\
\text { work and } \\
\text { submitted it }\end{array}$ & $\begin{array}{c}32 \\
(4.3 \%)\end{array}$ & $\begin{array}{c}198 \\
(26.9 \%)\end{array}$ & $\begin{array}{c}89 \\
(12.1 \%)\end{array}$ & $\begin{array}{c}201 \\
(27.3 \%)\end{array}$ & $\begin{array}{c}217 \\
(29.4 \%)\end{array}$ & 737 \\
\hline $\begin{array}{l}\text { I have helped } \\
\text { a friend by } \\
\text { writing an } \\
\text { assignment } \\
\text { for him/her }\end{array}$ & $\begin{array}{c}142 \\
(19.3 \%)\end{array}$ & $\begin{array}{c}333 \\
(45.3 \%)\end{array}$ & $\begin{array}{c}89 \\
(12.1 \%)\end{array}$ & $\begin{array}{c}80 \\
(10.9 \%)\end{array}$ & $\begin{array}{c}91 \\
(12.4 \%)\end{array}$ & 735 \\
\hline
\end{tabular}

Table 5 shows deviation from practising academic honesty regarding assignments. The highest percentage of deviation from academic honesty is $26.9 \%$ (Agreeing) to have modified another student's work and submitted it and 29.4\% (Strongly Disagreeing) to have copied answers from another student's work for an assignment and 31.9\% have copied from assignments submitted earlier by senior peers.

Table 6: Distribution of the respondents' opinions about deviation from practising academic honesty regarding Laboratory tests

\begin{tabular}{|c|c|c|c|c|c|c|}
\hline & & & & & & $=739$ \\
\hline Statement & 5 & 4 & 3 & 2 & 1 & Total \\
\hline & $\begin{array}{c}\text { SA } \\
\text { f(\%) }\end{array}$ & $\begin{array}{c}\mathrm{A} \\
\mathrm{f}(\%)\end{array}$ & $\begin{array}{c}\text { NAND } \\
\mathbf{f}(\%)\end{array}$ & $\begin{array}{c}D \\
f(\%)\end{array}$ & $\begin{array}{c}\text { SD } \\
\mathbf{f}(\%)\end{array}$ & \\
\hline $\begin{array}{l}\text { I have } \\
\text { reported a } \\
\text { laboratory } \\
\text { test or oral } \\
\text { dental } \\
\text { examination } \\
\text { as normal, } \\
\text { when it was } \\
\text { not done }\end{array}$ & $\begin{array}{c}43 \\
(5.9 \%)\end{array}$ & $\begin{array}{c}142 \\
(19.5 \%)\end{array}$ & $\begin{array}{c}83 \\
(11.4 \%)\end{array}$ & $\begin{array}{c}244 \\
(33.4 \%)\end{array}$ & $\begin{array}{c}218 \\
(29.9 \%)\end{array}$ & 730 \\
\hline $\begin{array}{l}\text { I have } \\
\text { falsified } \\
\text { (changed) } \\
\text { laboratory or } \\
\text { experimental } \\
\text { data }\end{array}$ & $\begin{array}{c}16 \\
(2.2 \%)\end{array}$ & $\begin{array}{c}90 \\
(12.3 \%)\end{array}$ & $\begin{array}{c}56 \\
(7.6 \%)\end{array}$ & $\begin{array}{c}256 \\
(34.9 \%)\end{array}$ & $\begin{array}{c}315 \\
(43 \%)\end{array}$ & 733 \\
\hline
\end{tabular}

Table 6 shows deviation from practising academic honesty regarding laboratory tests. Those responding by strongly agreeing (SA) was $5.9 \%$ and agreeing (A) was $19.5 \%$ to reporting a laboratory test or oral dental examination as normal, when it was not done. Those responding by strongly agreeing (SA) was $2.2 \%$ and agreeing (A) was $12.3 \%$ to have falsified laboratory or experimental data. The highest percentage of deviation from academic honesty is $43 \%$ 
strongly disagreeing (SD) to the statement. Respondents disagreeing $(33.4 \%)$ have reported a laboratory test or oral dental examination as normal, when it was not done.

Table 7: Distribution of the respondents' opinions about deviation from practising academic honesty regarding forging teacher's signature and grades

$\mathbf{n}=\mathbf{7 3 9}$

\begin{tabular}{|l|c|c|c|c|c|c|}
\hline Statement & $\mathbf{5}$ & $\mathbf{4}$ & $\mathbf{3}$ & $\mathbf{2}$ & $\mathbf{1}$ & Total \\
\cline { 2 - 6 } & $\begin{array}{c}\text { SA } \\
\mathbf{f}(\%)\end{array}$ & $\begin{array}{c}\mathbf{A} \\
\mathbf{f ( \% )}\end{array}$ & $\begin{array}{c}\text { NAND } \\
\mathbf{f}(\%)\end{array}$ & $\begin{array}{c}\mathbf{D} \\
\mathbf{f ( \% )}\end{array}$ & $\begin{array}{c}\text { SD } \\
\mathbf{f ( \% )}\end{array}$ & \\
\hline $\begin{array}{l}\text { I have forged } \\
\text { teacher's } \\
\text { signature on a } \\
\text { piece of work }\end{array}$ & $\begin{array}{c}26 \\
(3.6 \%)\end{array}$ & $\begin{array}{c}51 \\
(7.1 \%)\end{array}$ & $\begin{array}{c}52 \\
(7.2 \%)\end{array}$ & $\begin{array}{c}186 \\
(25.8 \%)\end{array}$ & $\begin{array}{c}406 \\
(56.3 \%)\end{array}$ & 721 \\
\hline $\begin{array}{l}\text { I have } \\
\text { changed } \\
\text { grades in the } \\
\text { record book }\end{array}$ & $\begin{array}{c}17 \\
(2.3 \%)\end{array}$ & $\begin{array}{c}40 \\
(5.5 \%)\end{array}$ & $\begin{array}{c}29 \\
(4 \%)\end{array}$ & $\begin{array}{c}182 \\
(24.8 \%)\end{array}$ & $\begin{array}{c}465 \\
(63.4 \%)\end{array}$ & 733 \\
\hline
\end{tabular}

Table 7 shows that a high percentage (56.3\%) of respondents strongly disagreed (SD) to having forged a teacher's signature on a piece of work. Similarly $63.4 \%$ respondents strongly disagreed (SD) to have changed grades in the record book. Those responding by strongly agreeing (SA) was $3.6 \%$ and agreeing (A) was 7.1\% to have forged teacher's signature on a piece of work. Those responding by strongly agreeing (SA) was $2.3 \%$ and agreeing (A) was $5.5 \%$ to have changed grades in the record book.

Table 8: Distribution of the respondents' opinions about deviation from practising academic honesty regarding written examinations

$\mathrm{n}=\mathbf{7 3 9}$

\begin{tabular}{|c|c|c|c|c|c|c|}
\hline \multirow[t]{2}{*}{ Statement } & 5 & 4 & 3 & 2 & 1 & \multirow[t]{2}{*}{ Total } \\
\hline & $\begin{array}{c}\text { SA } \\
\mathbf{f}(\%)\end{array}$ & $\begin{array}{c}\mathbf{A} \\
\mathbf{f}(\%)\end{array}$ & $\begin{array}{c}\text { NAND } \\
\mathbf{f}(\%)\end{array}$ & $\begin{array}{c}D \\
\mathbf{f}(\%)\end{array}$ & $\begin{array}{c}\text { SD } \\
\mathbf{f}(\%)\end{array}$ & \\
\hline $\begin{array}{l}\text { I have cheated } \\
\text { in examinations } \\
\text { using notes } \\
\text { taken into the } \\
\text { examination } \\
\end{array}$ & $\begin{array}{c}15 \\
(2 \%)\end{array}$ & $\begin{array}{c}65 \\
(8.8 \%)\end{array}$ & $\begin{array}{c}45 \\
(6.1 \%)\end{array}$ & $\begin{array}{c}190 \\
(25.9 \%)\end{array}$ & $\begin{array}{c}420 \\
(57.1 \%)\end{array}$ & 735 \\
\hline $\begin{array}{l}\text { I have copied } \\
\text { answers from } \\
\text { another student } \\
\text { during an } \\
\text { examination }\end{array}$ & $\begin{array}{c}57 \\
(7.7 \%)\end{array}$ & $\begin{array}{c}229 \\
(31 \%)\end{array}$ & $\begin{array}{c}105 \\
(14.2 \%)\end{array}$ & $\begin{array}{c}152 \\
(20.6 \%)\end{array}$ & $\begin{array}{c}196 \\
(26.5 \%)\end{array}$ & 739 \\
\hline $\begin{array}{l}\text { I have used } \\
\text { cheat sheets } \\
\text { during } \\
\text { examinations }\end{array}$ & $\begin{array}{c}28 \\
(3.8 \%)\end{array}$ & $\begin{array}{c}63 \\
(8.6 \%)\end{array}$ & $\begin{array}{c}42 \\
(5.7 \%)\end{array}$ & $\begin{array}{c}159 \\
(21.7 \%)\end{array}$ & $\begin{array}{c}441 \\
(60.2 \%)\end{array}$ & 733 \\
\hline
\end{tabular}

Table 8 shows the deviation from practising academic honesty regarding written examinations. $57.1 \%$ respondents said that they strongly disagreed (SD) having cheated in examinations using notes taken into the examination, while only $2 \%$ strongly agreed (SA) to have used notes taken into the examination. Using cheat sheets has a similar finding with $60 \%$ strongly disagreeing (SD) to the statement. Copying answers from another student has a lower percentage $(26.5 \%)$ strongly disagreeing (SD). Those responding by strongly agreeing (SA) was $2 \%$ and agreeing (A) was $8.8 \%$ to have cheated in examinations using notes taken into the examination. Those responding by strongly agreeing (SA) was $7.7 \%$ and agreeing (A) was $31 \%$ to have copied answers from another student during an examination. Those responding by strongly agreeing (SA) was $3.8 \%$ and agreeing (A) was $8.6 \%$ to have used cheat sheets during examinations.

Table 9: Distribution of the respondents' opinions about deviation from practising academic honesty regarding practical examinations

$\mathbf{n}=\mathbf{7 3 9}$

\begin{tabular}{|c|c|c|c|c|c|c|}
\hline \multirow[t]{2}{*}{ Statement } & 5 & 4 & 3 & 2 & 1 & \multirow[t]{2}{*}{ Total } \\
\hline & $\begin{array}{c}\text { SA } \\
\mathbf{f}(\%)\end{array}$ & $\begin{array}{c}\mathbf{A} \\
\mathbf{f}(\%)\end{array}$ & $\begin{array}{c}\text { NAND } \\
\text { f(\%) }\end{array}$ & $\begin{array}{c}D \\
\mathbf{f}(\%)\end{array}$ & $\begin{array}{l}\text { SD } \\
\mathbf{f}(\%)\end{array}$ & \\
\hline $\begin{array}{l}\text { I have changed } \\
\text { labels and slides } \\
\text { in an } \\
\text { OSPE/OSCE } \\
\text { examination }\end{array}$ & $\begin{array}{c}11 \\
(1.5 \%)\end{array}$ & $\begin{array}{c}65 \\
(8.9 \%)\end{array}$ & $\begin{array}{c}45 \\
(6.1 \%)\end{array}$ & $\begin{array}{c}178 \\
(24.3 \%)\end{array}$ & $\begin{array}{c}435 \\
(59.3 \%)\end{array}$ & 734 \\
\hline $\begin{array}{l}\text { I have talked to } \\
\text { another student } \\
\text { about an OSCE }\end{array}$ & $\begin{array}{c}63 \\
(9.6 \%)\end{array}$ & $\begin{array}{c}328 \\
(44.9 \%)\end{array}$ & $\begin{array}{c}84 \\
(11.1 \%)\end{array}$ & $\begin{array}{c}122 \\
(16.7 \%)\end{array}$ & $\begin{array}{c}133 \\
(18.2 \%)\end{array}$ & 730 \\
\hline $\begin{array}{l}\text { I have used } \\
\text { electronic } \\
\text { devices, such } \\
\text { as smart } \\
\text { phones and } \\
\text { tablets, without } \\
\text { permission }\end{array}$ & $\begin{array}{c}55 \\
(7.5 \%)\end{array}$ & $\begin{array}{c}218 \\
(29.6 \%)\end{array}$ & $\begin{array}{c}61 \\
(8.3 \%)\end{array}$ & $\begin{array}{c}140 \\
(19 \%)\end{array}$ & $\begin{array}{c}263 \\
(35.7 \%)\end{array}$ & 337 \\
\hline
\end{tabular}

Table 9 shows the deviation from practising academic honesty regarding practical examinations. Respondents said that they strongly disagreed $(59.3 \%)$ having cheated in OSPE/OSCE examinations, OSPE/OSCE. while only $1.5 \%$ strongly agreed to have changed labels and slides. Talking to another student during practical examinations has a similar finding with $44.9 \%$ agreeing of the statement. Using electronic devices without permission has a lower percentage $(7.5 \%)$ strongly agreeing.

\section{Discussion}

Copying at class tests and university examinations, copying/cooking up records and case sheets, forging teachers' signatures in records, altering marks entered in papers, giving proxy for attendance at teaching sessions, all seem to be common practice for undergraduate students in Indian medical colleges (Gitanjali B, 2004). Use of cell phones without permission has been reported in a study by Rowland ML \& Srisukho K (2009) and by Ward C \&Yates D (2014). Hafeez K, Khan M, Jawaid M \& Haroon S (2013) stated that in their study they found that $55.1 \%$ cheated at least once in their medical college. 
Asking a friend to sign proxy attendance for oneself seems to be a very common phenomenon among students in dental colleges. Most of the students $(61.7 \%)$ said they disagreed (SD and D) to asking a friend to sign proxy attendance for them. Only $28.9 \%$ (SA and A) said they signed proxy attendance. Also the highest percentage $(62 \%)$ claimed to disagree (SD and D) to signing proxy attendance at for a friend who did not attend the class. Tanawattananacharoen $\mathrm{S} \&$ Nimnuan C (2009) have reported $41.3 \%$ and $49 \%$ students not attending class but asking a friend to sign proxy attendance and signing a proxy attendance for a friend respectively.

Students tend to remain absent from classes for various reasons. A high percentage of respondents claimed that they did not fake illness to justify their absence (49.7\%). Similarly a high percentage of respondents claimed that they did not remain absent for class without any genuine cause (29.6\%). The highest percentage $(62.1 \%)$ of respondents claimed that they did not submit fake medical certificates to justify illness. Tanawattananacharoen $\mathrm{S} \&$ Nimnuan C(2009) have reported students fake illness like food poisoning to remain absent from class.

Koletsi-Kounari H, Polychronopoulou A, Reppa C \& Teplitsky PE (2011) stated that students reported to have missed lectures frequently. A high percentage of respondents claimed that they stayed in the dental college campus during academic hours $(45.6 \%)$. A high number of respondents (43.4\%) strongly disagreed to the fact that they left before class finished without permission.

To complete assignments on time, as the workload is high in dental colleges, copying from others and plagiarism may occur. The highest percentage of deviation from academic honesty is $43 \%$ (Agreeing) practising plagiarism, 33.4\% (Agreeing) to have copied answers form another student's work for an assignment and $31.9 \%$ have copied form assignments submitted earlier by senior peers. Tanawattananacharoen S \& Nimnuan C(2009) have reported students have modified a friend's work and submitted it.

There is a trend to alter another student's written work and submit it. The highest percentage of deviation from academic honesty is $26.9 \%$ (Agreeing) to have modified another student's work and submitted it and $29.4 \%$ (Strongly Disagreeing) to have copied answers form another student's work for an assignment and 31.9\% have copied form assignments submitted earlier by senior peers. KoletsiKounari H, Polychronopoulou A, Reppa C \& Teplitsky PE (2011) stated that students reported to have an entire essay assignment. Tanawattananacharoen S \& Nimnuan $\mathrm{C}$ have reported 15.25 students submitting work submitted by a senior student the previous year.

The highest percentage of deviation from academic honesty is $12.3 \%$ (Agreeing) to have falsified laboratory or experimental data and submitted it and 43\% (Strongly Disagreeing) to the statement. Respondents disagreeing (33.4\%) have reported a laboratory test or oral dental examination as normal, when it was not done. During clinical rotations, students are observed to write fake histories and examinations which they have not asked or performed (Hafeez K, Khan M, Jawaid M \& Haroon S, 2013).

Due to various time limits and work overload there seems to be some students who resort to forging teacher's signature and changing grades in record keeping. A high percentage (56.3\%) of respondents strongly disagreed to having forged a teacher's signature on a piece of work. Similarly $63.4 \%$ respondents strongly disagreed to have changed grades in the record book. Koletsi-Kounari H, Polychronopoulou A, Reppa C \& Teplitsky PE (2011) have said that students have forged a faculty signature on a patient chart. Tanawattananacharoen $\mathrm{S}$ \& Nimnuan Chave reported that a percentage of $5.3 \%$ medical students have forged staff's signature on a piece of work. Andrews KG, Smith LA, Henzi D \& Demps E (2007) reported an incresded incidence in forging a teacher's signature. Some of the students also accepted to have forged a teacher's signature (16.9\%), according to Hafeez K, Khan M, Jawaid M \& Haroon S (2013).

This is an area of concern in all countries. Cheating in exams seems to be wide spread. Respondents claim to strongly disagree $(57.1 \%)$ having cheated in examinations using notes taken into the examination, while only $2 \%$ strongly agree to have used notes taken into the examination. Using cheat sheets has a similar finding with $60 \%$ strongly disagreeing o the statement. Copying answers from another student has a lower percentage (26.5\%) strongly disagreeing. Koletsi-Kounari H, Polychronopoulou A, Reppa C \& Teplitsky PE (2011) have said that students have Cheated on a written exam using "crib notes". Tanawattananacharoen S \& Nimnuan $\mathrm{C}$ have reported $33.2 \%$ students copying answers from a friend in an examination.

Respondents claim to strongly disagree (59.3\%) having cheated in OSPE/OSCE examinations, OSPE/OSCE while only $1.5 \%$ strongly agreed to have changed labels and slides. Talking to another student during practical examinations has a similar finding with $44.9 \%$ disagreeing o the statement. Using electronic devices without permission has a lower percentage $(7.5 \%)$ strongly disagreeing. According to Hafeez K, Khan M, Jawaid M \& Haroon S (2013), during OSCE most of the students share common stations and students may pass information to their colleagues. In their study, $83.6 \%$ of students accepted that they tell their friends about the questions which were asked in first shift.

\section{Conclusion}

The causes of the students' deviation from practising academic honesty were that $66.1 \%$ of the students claimed that some assignments are burdensome to complete in terms of time, $61.7 \%$ said that they experienced burnout from work (content) overload, and $58.6 \%$ had pressure from parents to do well academically.

We need to improve our educational system, formally add professional sessions in the curriculum. The study recommended that Dental Colleges and Dental Educators 
must be aware stay of these dimensions of academic honesty, and they must ensure that dental students, faculty members, and staff are ethical caretakers of this ethical practise. Dental colleges must either create or reinforce an ethical culture on campus that should include students, faculty members, and staff; increase or modify the teaching of ethics to all students.

\section{References}

1. Al-Dwairi ZN \& Al-Waheidi EM (2004), Cheating Behaviors of Dental Students, Journal of Dental Education, November 2004, Volume 68, Number 11, pp 1192-1195, Abstract

2. Anderson RE \&Obenshain SS (1994), Cheating by students: findings, reflections, and remedies, Academic Medicine, 1994 May; Volume 69, number 5,pp 323-32

3. Andrews KG, Smith LA, Henzi D \&Demps E (2007), Faculty and Student Perceptions of Academic Integrity at U.S. and Canadian Dental Schools; Journal of Dental Education, August 1, 2007 vol. 71 no. 8, pp 1027-1039

4. Anderson RE \&Obenshain, SS (1994) Cheating by students: findings, reflections and remedies, Academic Medicine, 69, Abstract

5. Asokan S, Baby John JB, Janani D, Jessy P, Kavya S \& Sharma K (2013) Attitudes of Students and Teachers on Cheating Behaviors: Descriptive Cross- Sectional Study at Six Dental Colleges in India, Journal of Dental Education, October 2013,pp 1379- 1383

6. Baldwin DC Jr, Daugherty SR, Rowley BD \& Schwartz $\mathrm{R}(1996)$ Cheating in medical school: a survey of second year students at 31 schools, Academic Medicine, 71, pp. 267-273

7. Brondani MA \&Rossoff LP (2010), The Hot Seat Experience: A Multifaceted approach to the Teaching Ethics in a Dental Curriculum, Journal of Dental Education, Volume 69, Number 2, February 2005, pp 213-224

8. Chambers DW (2010), The Role of Empathy in Ethics,Journal of Dental Education,December 2010, Volume 74, Number 12, pp 1275-1281

9. Cederberg, RA \&Valenza JA (2012) Ethics and the Electronic Health record in Dental school clinics. Journal of Dental Education, May 2012, Volume 76, Number 5, pp 584- 588

10. Christie C, Bowen D \&Paarmann C (2007), Effectiveness of Faculty Training to Enhance Clinical Evaluation of Student Competence in Ethical Reasoning and Professionalism, Journal of Dental Education, Volume 71, Number 8, pp 1048-1057

11. Douglas College (2016), Academic Integrity Policy, http://www.douglascollege.ca/ /media/E9051B834E5 742CB90480DA897A6B7AB.ashx?la=en, Accessed: March 28, 2017

12. Graham BS, Knight GW \&Linda Graham (2016),
Dental Student Academic Integrity in U.S. Dental Schools: Current Status and Recommendations for Enhancement, Journal of Dental Education, January 1, 2016 vol. 80 no. $1,5-13$

13. Gitanjali B (2004), Academic dishonesty in Indian medical colleges, Journal of Postgraduate Medicine, December 2004, Vol 50, Issue 4, pp 281-284

14. Hafeez K, Khan M, Jawaid M \&Haroon S (2013), Academic misconduct among students in medical colleges of Karachi, Pakistan, Pak J Med Sci. 2013 May-Jun; 29(3):pp 699-702.

15. Hutchins B \& Cobb S (2008), When Will We Be Ready for Academic Integrity? Journal of Dental Education, Volume 72, Number 3, March 2008, pp 359-363

16. Koerber A, Botto RW, Pendleton DD, Albazzaz MB \&Doshi SJ (2010), Enhancing Ethical Behavior: Views of Students, Administrators, and Faculty, Journal of Dental Education, Volume 69, Number 2, February 2005, pp 213-224, Abstract

17. Koletsi-Kounari H, Polychronopoulou A, Reppa C \&Teplitsky PE (2011), Penalties for Academic Dishonesty in a Greek Dental School Environment, Journal of Dental Education, October 2011 , pp 13831389

18. Kulsoom G, Ghulam R \&Hamna A (2014), Selfreported attitudes and behaviours of medical students in Pakistan regarding academic misconduct: a crosssectional study, 64, BMC Medical Ethics 2014, 15:43 Page 2 of 14 http://www.biomedcentral.com/1472$6939 / 15 / 43$

19. Kusnoor AV \& Ruth Falik R (2013), Cheating in Medical School: The Unacknowledged Ailment, Southern Medical Journal, Volume 106, Number 8, August 2013, pp 479-483

20. McCabe DL (2007), The fundamental values for academic integrity. Center for Academic Integrity, Duke University, www.academicintegrity.org/icai/assets/ FVproject.pdf,Accessed: March 28, 2017

21. Michigan State University, College of Human Medicine, Academic Honesty, www.msu.edu/unit/ o mbud/academic-integrity/index.html, www. humanmedicine.msu.edu/current/documents/MSRRan dAddendum.pdf

Accessed: March 29, 2017

22. Muhney KA (2010), Allied Dental and Dental Educators' Perceptions of and Reporting Practices on Academic Dishonesty, Journal of Dental Education, Volume 74, Number 11, November 2010, pp 1214-1219

23. Nash DA (2010), Ethics, Empathy, and the Education of Dentists, Journal of Dental Education, June 2010, Volume 74, Number 6, pp 567-578

24. Nash DA (2010), Letter to Editor: Thee Role of Empathy in Ethics, Journal of Dental Education, December 2010, Volume 74, Number 12, pp 1275-1281 
25. Nonis S, Swift CO: An examination of the relationship between academic dishonesty and workplace dishonesty: a Multicampus investigation. Journal Educational Business 2001, 77(2):69-77.)

26. Patel-Bhakta HG, KB Muzzin KB, DeWald JP, Campbell PR \&Buschang PH (2014), Attitudes Towards Students Who Plagiarize: A Dental Hygiene Faculty Perspective; Journal of Dental Education, January 2014, pp 131-145

27. Rennie, S, Crosby, J,(2001) Are "tomorrow's doctors" honest? Questionnaire study exploring medical students' attitudes and reported behaviour on academic misconduct, BMJ 2001; 322 doi: http://dx.doi.org/ 10.1136/, bmj.322.7281.274 (Published 03 February 2001), BMJ 2001;322:274

28. Rowland ML \&Srisukho K (2009), Dental Students ${ }^{\text {ee }}$ and Faculty Members ${ }^{\text {ee }}$ Perceptions of Incivility in the Classroom; January 2009, Journal of Dental Education, Volume 73, Number 1, pp 119-126
29. Student Code of Academic Integrity and Professionalism (2010), New York Medical College, http://www.nymc.edu/UniversityPolicies., Accessed: March 28, 2017

30. Tanawattananacharoen S \&Nimnuan C (2009), Academic misconduct among medical students, South East Asian Journal of Medical Education, 2009, Volume 3, Number 1, pp 8-13

24. Ward C \&Yates D (2014), Civility In The University Classroom: An Opportunity For Faculty To Set Expectations, Contemporary Issues In Education Research, Second Quarter 2014 Volume 7, Number 2, pp 165-170 